SIVAN MAUER

Relação entre Temperamentos Afetivos e Diagnósticos

Psiquiátricos em Idosos: Um Estudo Caso-Controle

SÃO PAULO

2020 


\section{SIVAN MAUER}

Relação entre Temperamentos Afetivos e Diagnósticos

Psiquiátricos em Idosos: Um Estudo Caso-Controle

Tese apresentada à Faculdade de Medicina da Universidade de São Paulo para obtenção do título de Doutor em Ciências

Programa de Psiquiatria

Orientador: Prof. Dr. Ivan Aprahamian

\section{SÃO PAULO}

2020 


\section{Dados Internacionais de Catalogação na Publicação (CIP)}

Preparada pela Biblioteca da

Faculdade de Medicina da Universidade de São Paulo

Creproduçāo autorizada pelo autor

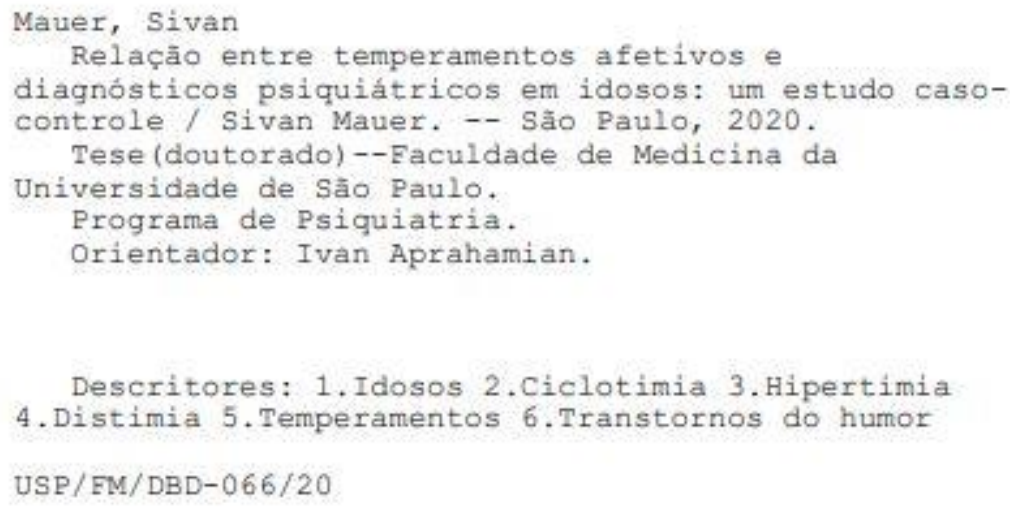

Responsável: Erinalva da Conceição Batista, CRB-8 6755 


\section{DEDICATÓRIA}

Esta tese é dedicada à minha esposa, Vanessa, que tem sido uma constante fonte de apoio e incentivo durante os desafios do meu percurso acadêmico e da minha vida. Sou verdadeiramente grato por tê-la em minha vida. 


\section{Agradecimentos}

Quando iniciei este doutorado vim em busca de mais conhecimento para tentar entender ainda mais as doenças psiquiátricas e assim dar segmento a minha jornada iniciada ha cerca de uma década em Boston, com meu mentor e amigo Nassir Ghaemi. Assim devo agradecer há inúmeras pessoas que fizeram parte desta trajetória.

Em primeiro lugar agradeço ao meu orientador Dr. Ivan Aprahamian pela oportunidade, apoio e constante disposição em dividir comigo seu tempo e conhecimento.

Gostaria de agradecer imensamente a toda as equipes do AME Dra. Jandira Mansur, localizado na cidade de São Paulo e ao ambulatório de Reumatologia da Universidade Mackenzie em Curitiba, que me acolheram e assim possibilitaram grande parte da coleta de dados utilizados nesta pesquisa. Neste sentido também gostaria de agradecer a todos os colegas do Ambulatório de Alterações Comportamentais em Idosos do HCFMUSP (ACID) e ao Ambulatório de Geriatria da Faculdade de Medicina de Jundiaí.

Um último agradecimento especial a todos os pacientes que se dispuseram a participar deste estudo. 


\section{SUMÁRIO}

\section{Lista de figuras}

\section{Lista de tabelas}

\section{Resumo}

\section{Abstract}

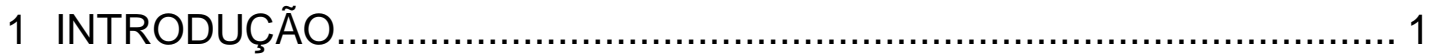

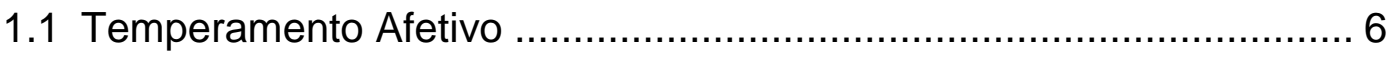

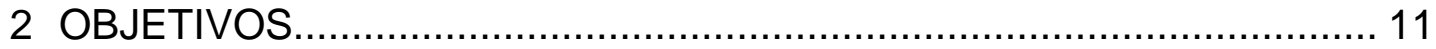

2.1 Objetivo Geral.................................................................... 11

2.2 Objetivos Específicos ............................................................. 11

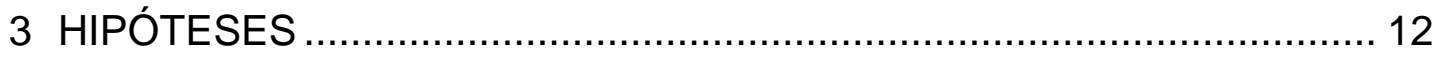

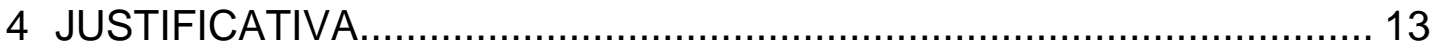

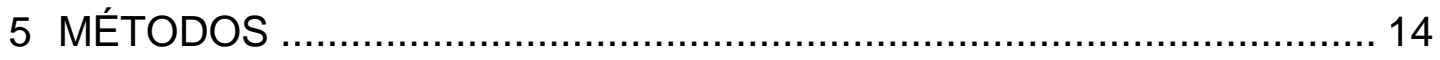

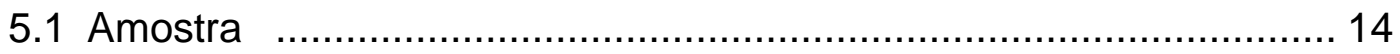

5.2 Instrumentos de Avaliação Clínica........................................ 16

5.3 Consentimento Informado............................................ 17

5.4 Análise Estatística ................................................................ 17

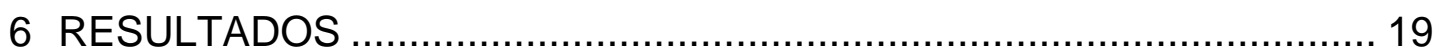

6.1 Medida de associação entre diagnósticos psiquiátricos (TAB e depressão) e temperamentos. ............................................. 24

6.1.1 TAB e Temperamentos .................................................. 24

6.1.2 Depressão e Temperamentos.............................................. 25

6.1.3 Desfechos secundários ................................................... 25

7 DISCUSSÃO

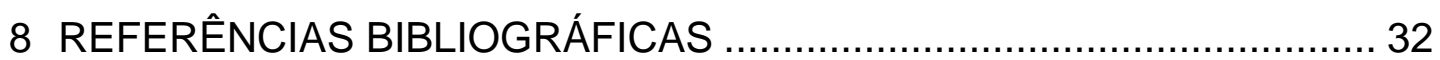




\section{LISTA DE FIGURAS}

Figura 1: Fluxograma da busca de artigos da revisão sistemática ............. 10

Figura 2: Fluxograma de inclusão de pacientes....................................... 15 


\section{LISTA DE TABELAS}

Tabela 1: Características sociodemográficas e antecedentes psiquiátricos da

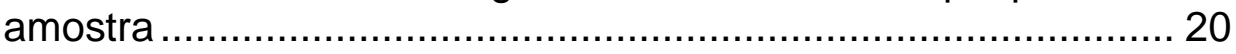

Tabela 2: Antecedentes psiquiátricos familiares …………..................... 21

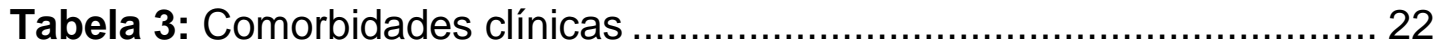

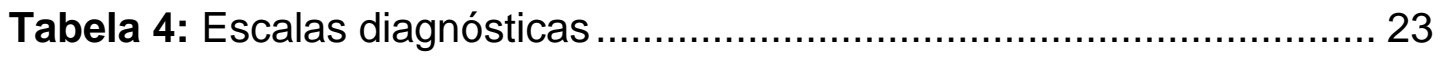

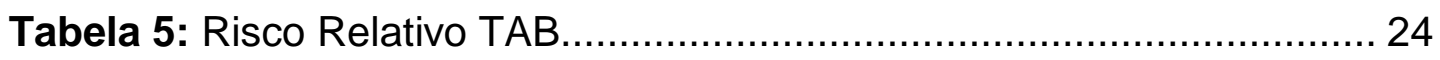

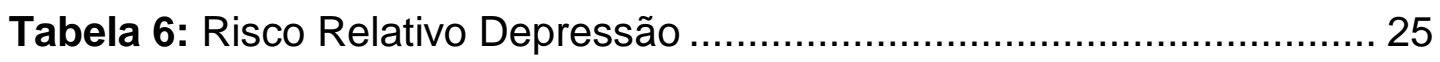




\section{RESUMO}

Mauer S. Relação entre temperamentos afetivos e diagnósticos psiquiátricos em idosos: um estudo caso-controle [tese]. São Paulo: Faculdade de Medicina, Universidade de São Paulo; 2020.

Um conceito pertinente na avalição do curso dos transtornos do humor, e muitas vezes ignorado pela psiquiatria, é o conceito de temperamentos afetivos. Emil Kraepelin descreveu os quatro elementos básicos (depressivo, maníaco, ciclotímico e irritável), os quais ele considerava formas subclínicas e possíveis precursores dos transtornos afetivos, e que tinham raízes na adolescência. O conceito de temperamento afetivo foi perdido no século XX com 0 crescimento das teorias psicanalíticas, onde o conceito de personalidade era visto sob a lente psicológica ao invés de biológica. Baseado neste contexto, os objetivos principais deste estudo foram identificar e comparar a frequência dos três principais tipos de temperamento (distimia, hipertimia, ciclotimia), além da associação dos diagnósticos e os três tipos de temperamento. $O$ estudo foi realizado com pacientes idosos diagnosticados com transtorno afetivo bipolar (TAB) e depressão maior, e em pacientes Controles. Para isto foram avaliados 22 casos de transtorno afetivo bipolar, 50 casos de depressão maior e 100 pacientes controles. Para este estudo foram usados os seguintes instrumentos de avaliação clínica: Mini Exame do Estado Mental, HAMD17, Hamilton para ansiedade, YMRS e TEMPS-A. Os pacientes do grupo com TAB apresentaram a maior incidência de hipertimia $(59,1 \%)$, os pacientes com depressão maior apresentaram uma incidência maior também de hipertimia (48\%). Em relação a análise de associação entre TAB e depressão maior e os três principais temperamentos, a medida usada foi o risco relativo (RR). Pacientes distímicos tiveram uma chance aumentada para o diagnóstico de TAB ( $R R=3,6,95 \%$ IC 1,1-12,4), os pacientes hipertímicos apresentaram um RR estatisticamente significante, mas protetivo. Já os pacientes com depressão maior apresentaram uma chance maior de diagnóstico entre os pacientes ciclotímicos ( $R R=3,2,95 \%$ IC 1,7-6,0) e uma chance menor entre os pacientes hipertímicos (RR=0,6, 95\% IC 0,4$0,8)$. Foram analisadas as relações de alguns desfechos secundários (tentativa de suicídio, abuso na infância, abuso sexual e automutilação). Podese concluir neste estudo que sujeitos com temperamento ciclotímico tem um risco maior de serem diagnosticados tanto com TAB quanto com depressão maior, quando comparados aos Controles. Entretanto, pacientes com temperamento hipertímico possuem um risco diminuído de apresentarem diagnóstico de depressão maior.

Descritores: Idosos; Ciclotimia; Hipertimia; Distimia; Temperamento; Transtornos do humor. 


\begin{abstract}
Mauer S. Relationship between affective temperament and psychiatric diagnosis in older adults: a case-control study [thesis]. São Paulo: "Faculdade de Medicina, Universidade de São Paulo"; 2020.

A relevant concept in assessing the course of mood disorders, and often ignored by psychiatry, is the concept of affective temperaments. Emil Kraepelin described the four basic elements (depressive, manic, cyclothymic and irritable), which he considered subclinical forms and possible precursors of affective disorders, and which had their roots in adolescence. The concept of affective temperament was lost in the twentieth century with the growth of psychoanalytic theories, where the concept of personality was seen through a psychological rather than a biological concept. Based on this context, the main aims of this study were to identify and compare the frequency of the three main types of temperament (dysthymia, hyperthymia, cyclothymia), in addition to the association of diagnoses and the three types of temperament The study was conducted with older adults diagnosed with bipolar disorder (BD) and major depression, and in Control patients. For this, 22 cases of bipolar disorder, 50 cases of major depression and 100 control patients were evaluated. For this study, the following rating scales were used: Mini Mental State Examination, HAMD17, Hamilton Scale for anxiety, YMRS and TEMPS-A. Patients in the group with BD had the highest incidence of hyperthymia $(59.1 \%)$, patients with major depression also had a higher incidence of hyperthymia (48\%). Regarding the analysis of the association between BD and major depression and the three main temperaments, the measure used was the relative risk $(R R)$. Dysthymic patients had an increased chance of diagnosing $B D(R R=$ $3.6,95 \% \mathrm{Cl} 1.1-12.4)$, hyperthymic patients had a statistically significant but protective RR. Patients with major depression had a greater chance of diagnosis among cyclothymic patients $(\mathrm{RR}=3.2,95 \% \mathrm{Cl} 1.7-6.0)$ and a lower chance among hyperthymic patients ( $R R=0.6,95 \% \mathrm{Cl} 0.4-0.8)$. The relationships of some secondary outcomes (suicide attempt, childhood abuse, sexual abuse and self-harm) were analyzed. It can be concluded in this study that subjects with cyclothymic temperament have a higher risk of being diagnosed with both BD and major depression, when compared to Controls. However, patients with hyperthymic temperament have a reduced risk of having a diagnosis of major depression.
\end{abstract}

Descriptors: Aged; Cyclothymia; Hyperthymia; Dysthymia; Temperament; Mood disorders. 


\section{INTRODUÇÃO}

No século XVIII existia apenas uma doença psiquiátrica: a insanidade. O diagnóstico de insanidade significa o que clinicamente hoje chamamos de psicose ou, o que na linguagem leiga se entende como "loucura"1. A criação da palavra psiquiatria não foi acidental. John Cristian Reil, um proeminente médico, anatomista, fisiologista e professor alemão das universidades de Halle e de Berlin, baseando-se nas suas leituras de Kant, formou novos conceitos dos mecanismos das doenças mentais e elaborou o termo em seu tratado em 1808. Neste tratado foi definido ao menos duas razões essenciais para se estabelecer uma nova disciplina médica: a continuidade da Psique e do corpo e a inseparabilidade entre psiquiatria e medicina ${ }^{2,3}$.

Wilhelm Griesinger, outro psiquiatra alemão, definiu pela primeira vez em 1867, a doença mental como uma doença do cérebro ${ }^{4}$. Karl Ludwing Kahlbaum foi um dos grandes colaboradores do estudo do curso clínico descritivo longitudinal em psicopatologia, fazendo importantes contribuições ao conceito de transtornos afetivos recorrentes, além de desenvolver o termo catatonia ${ }^{5}$. Kraepelin deu continuidade a esta abordagem, tendo como um dos seus focos a sistematização dos temperamentos afetivos. O conceito de temperamento faz parte da noção de doença maníaco depressiva de Kraepelin, o qual tinha uma visão muito moderna de doença afetiva. Sua hipótese era que as recorrências surgiam de disposições duradouras de depressão, ciclotimia e mania ${ }^{6}$. É importante esclarecer que a doença maníaco depressiva não é sinônimo de transtorno afetivo bipolar. O conceito 
de doença maníaco depressiva foi dividido oficialmente em 1980 com o advento da terceira edição do Manual Diagnóstico e Estatístico de Transtornos Mentais (DSM-III), da Associação de Psiquiatria Americana, em transtorno afetivo bipolar e depressão maior. Esta noção é diferente do que Karl Leonhard propôs nos anos 50, quando o conceito de doença maníaco depressiva era composto de transtorno afetivo bipolar e depressão unipolar recorrente psicótica ${ }^{7}$. Em resumo o que ocorreu, com o advento do DSM-III, foi uma substituição do conceito amplo de doença maníaco depressiva pelo transtorno afetivo bipolar, e uma grande variedade de sintomas depressivos nomeados por transtorno depressivo maior. Desde então existe um conceito diagnóstico bastante estreito de transtorno bipolar, o que difere da doença maníaco depressiva. Além disso, o transtorno afetivo bipolar é definido pela polaridade, ou seja, pela existência ou não de quadro maníaco. A doença maníaco depressiva é definida pela recorrência de episódios, independentemente da polaridade. Por exemplo, cem episódios depressivos ou cem episódios maníacos podem determinar o diagnóstico de doença maníaco depressivo. Aqui o tipo de polo é irrelevante, o que é relevante é a recorrência. Doença maníaco depressiva significa a associação de transtorno bipolar e transtorno depressivo maior. Normalmente, é referido que o DSM, a partir de terceira edição, passou a ser neo-Kraepeliniano. Porém, isto não é real, tendo em vista que ele passou a ser neo-Leonhardiano ${ }^{8}$.

Desde então o consenso neo-Leonhardiano do DSM-III se manteve. Apenas dois pesquisadores se contrapuseram a esta ideia: Hagop Akiskal, nos EUA, e Athanasios Koukopoulos, na Europa ${ }^{9}$. Akiskal começou seus 
estudos na década de 70, no primeiro serviço especializado em transtornos do humor dos EUA. Este identificou vários pacientes que ficavam entre bipolares e unipolares. Akiskal propôs que se mantivesse a dicotomia bipolar/unipolar, mas que ampliasse a categoria bipolar incluindo o espectro bipolar. Neste espectro foi incluído pacientes com depressões com apresentações atípicas e temperamentos afetivos. Akiskal também apresentou uma ideia importante para ampliar o conceito de transtorno bipolar tipo I, no DSM-III, e uma década depois o transtorno bipolar tipo II foi aceito, permitindo que episódios maníacos moderados chamados de hipomania, ocorressem conjuntamente com depressões recorrentes. $\mathrm{O}$ aspecto mais original que Akiskal acrescentou além da noção de espectro bipolar foi o do conceito de temperamentos afetivos. Estes temperamentos são genéticos e relacionados ao transtorno bipolar ou a depressão unipolar grave, ocorrendo em ao menos metade dos pacientes com transtorno bipolar ${ }^{10}$. Já Koukopoulos não conseguia comprovar o conceito unipolar/bipolar, principalmente pela resposta ao tratamento, pois muitos pacientes unipolares não respondiam adequadamente aos antidepressivos. Além disto, Koukopoulos identificou que muitos pacientes depressivos tinham sintomas maníacos e que muito pacientes maníacos tinham sintomas depressivos, ou seja os estados mistos eram muito mais frequentes que os estados puros polarizados entre depressivos ou maníacos ${ }^{11}$.

O diagnóstico psiquiátrico é um desafio em todas as faixas etárias, mas nos extremos como crianças e idosos, ele é ainda maior. Nos idosos, isto se deve a alguns fatores como dificuldade na identificação do curso longitudinal 
das doenças psiquiátricas, grande número de comorbidades clínicas e psiquiátricas, polifarmácia e a sobreposição de sintomas entre os vários diagnósticos psiquiátricos, como quadros demenciais e episódios depressivos. Além disso, há um forte estigma geral, entre a população leiga e até mesmo médica, de que o envelhecimento traz consigo sintomas como tristeza e esquecimento. Isto torna factível um cenário no qual o paciente idoso ignore sua mudança afetiva. Aliado a isso, semiologicamente, o idoso refere menos suas queixas globalmente. Estes fatores abrem algumas discussões em relação ao crescimento da prevalência dos transtornos mentais nesta faixa etária. Poucos estudos tratam da prevalência de transtornos mentais em idosos.

Alguns estudos mostram uma tendência de diminuição destes transtornos, mas a maioria deles apresentam problemas metodológicos, como amostras pequenas ${ }^{12,13}$. Um recente estudo brasileiro confirma a tendência da redução dos transtornos mentais nos idosos, porém concluiu-se também que em relação ao transtornos do humor não existe uma redução, mantendose ao menos nos mesmos patamares que na população adulta mais jovem ${ }^{14}$. Os transtornos do humor, como os transtornos bipolares e a depressão maior, em geral são os diagnósticos mais frequentes nesta população, junto com os quadros demenciais. As principais dificuldades na população idosa são a classificação, o diagnóstico (principalmente pela sobreposição de sintomas) e consequentemente o tratamento. Assim, é de extrema importância a identificação dos chamados validadores diagnósticos 
estabelecidos por Robins e Guze, que são: sintomas, história familiar, curso da doença, resposta ao tratamento e marcadores biológicos ${ }^{15}$.

Esta abordagem se contrapõe a usada pelo DSM, na qual foca quase que exclusivamente em experiência e julgamento clínico ${ }^{4}$. Entre as diversas consequências deste método de abordagem pelo sistema DSM, podemos verificar que a grande maioria dos diagnósticos não tem validade científica. Assim, constata-se que o uso da polifarmácia, por exemplo, é resultado da abordagem sintomatológica, ao invés do tratamento orientado na abordagem da doença $a^{16,17}$.

A avaliação do curso nos transtornos psiquiátricos nos idosos é de extrema relevância e decisiva para 0 correto diagnóstico e, consequentemente, no tratamento destas afecções, pois é nesta população que podemos observar e entender os vários anos de sintomatologia ou, muitas vezes, os períodos sem sintomas. Os exemplos mais comuns são os episódios depressivos recorrentes, os episódios depressivos ditos de início tardio (após os 50 anos) e os quadros de humor relacionados às questões vasculares, principalmente os episódios depressivos (talvez o mais característico desta faixa etária). Nos quadros vasculares os exames de imagem serviriam como marcadores biológicos, algo que em outras faixas etárias até o momento não se tem.

Um conceito pertinente na avalição do curso, e muitas vezes ignorado pela psiquiatria, é o conceito de temperamentos afetivos. Este conceito foi descrito originalmente na Grécia antiga por Hipócrates, onde melancolia era 
vista como excesso de bile negra e mania como excesso de bile amarela ${ }^{18}$. Areteu da Capadócia foi o primeiro autor a relacionar mania e melancolia na mesma doença, além de descrever ciclotimia como a alternância de fases depressivas e maníacas ${ }^{19}$. Emil Kraepelin descreveu os quatro elementos básicos (depressivo, maníaco, ciclotímico e irritável), os quais ele considerava formas subclínicas e possíveis precursores dos transtornos afetivos e que tinham raízes na adolescência ${ }^{20}$. Contudo, Ernest Kretshmer foi quem melhor sistematizou o conceito de temperamento descrevendo hipertimia pela primeira vez, uma vez que ciclotimia e distimia já haviam sido introduzidos na psiquiatria alemã e francesa no século $X \mid X^{21,22}$.

\subsection{Temperamento Afetivo}

O conceito de temperamento afetivo foi perdido no século $X X$ com o crescimento das teorias psicanalíticas, onde o conceito de personalidade era visto sob a lente psicológica ao invés de biológica, e relacionada a conceitos psicanalíticos de desenvolvimento psicológicos ${ }^{23}$. Alguns termos como temperamento e personalidade têm sido utilizados quase como sinônimos para classificar condições de curso crônico em psiquiatria. No desenvolvimento do DSM-III, a personalidade depressiva foi descrita como uma forma de doença afetiva, renomeada como distimia e inserida sob Eixo I, e não sob Eixo II, que incluem os transtornos de personalidade. $\mathrm{O}$ mesmo ocorreu com o conceito de personalidade cicloide, que passou a se chamar ciclotimia. Já o conceito de hipertimia foi completamente esquecido $6,24,25,26$. 
As definições dos três principais tipos de temperamentos afetivos podem ser conceitualizadas da seguinte forma: hipertimia, distimia e ciclotimia. Hipertimia envolve sintomas maníacos leves como parte da personalidade. Estes sujeitos tem um nível de energia mais alto, menos necessidade de sono, libido mais alta, são muito sociáveis e suscetíveis ao abuso de álcool e outras drogas ${ }^{27,28}$. Distimia representa sintomas depressivos suaves como parte da personalidade. O paciente tem entre suas características um baixo nível de energia, necessidade de mais horas de sono que a média em geral, libido diminuída, maior inclinação a ter preocupações relacionadas a falhas pessoais ${ }^{6,29}$. Ciclotimia é uma constante alternância entre sintomas maníacos suaves e estados depressivos diários, e que acabam não satisfazendo os critérios temporais para síndromes afetivas completas. São pessoas com características como labilidade, raiva e irritabilidade, além de apresentarem dificuldades atencionais ${ }^{6}$.

Os temperamentos afetivos são conceitualizados como parte do espectro das doenças afetivas. Este entendimento é embasado em estudos familiares, genéticos e clínicos. O espectro afetivo é descrito como um continuo entre ciclotimia, transtorno bipolar tipo I e tipo II, assim como depressão subsindrômica, depressão menor e depressão maior ${ }^{30,31}$.

Os temperamentos têm uma origem genética e biológica, embasados no curso continuo entre os temperamentos afetivos e transtornos do humor, os quais também tem uma alta hereditariedade. Alguns estudos têm demonstrado que parentes saudáveis de probandos de pacientes com 
transtorno afetivo bipolar têm um alto grau de desregulação temperamental, quando comparados a controles saudáveis ${ }^{30}$. Estudos genéticos familiares identificaram uma forte relação do temperamento ciclotímico e menos do temperamento hipertímico entre parentes de primeiro grau de pacientes com transtorno bipolar tipo ${ }^{32}$. Uma questão levantada a partir deste contínuo é o conceito de remissão nos quadros de humor recorrentes. Remissão é um conceito de extrema importância para o curso da doença, a qual estaria associada com o conceito de temperamento. O conceito de remissão é reconhecido como o melhor desfecho para o tratamento dos transtornos do humor ou como a ausência ou presença mínima de sintomas de mania e depressão por ao menos uma semana ${ }^{33,34}$. No entanto, um significado preciso e que constituiria um estado saudável para pacientes com transtornos do humor ainda não foi definido. Tenta-se estabelecer parâmetros claros como uma pontuação na Escala de Depressão de Hamilton 17 itens (HAMD-17) com menos de 5 pontos, ou na Escala de Depressão de Montgomery and Asberg (MADRS) menor que 5 pontos ou na Escala Breve de Avaliação Psiquiátrica (BDRS) com menos de 8 pontos para depressão e a Escala de Avaliação de Mania de Young (YMRS) com menos ou igual a 8 pontos para mania ${ }^{35}$. No entanto, um estudo demonstrou que apenas $25 \%$ dos pacientes que atingiram os parâmetros para remissão se sentiam dessa forma ${ }^{36,37}$. Um estudo recente mostrou que pacientes diagnosticados com depressão maior e temperamento afetivo do tipo de esquiva de dano (definido por Cloninger, correspondente ao temperamento distímico), tratados com antidepressivo, apresentaram mais dificuldades de chegar a remissão ${ }^{38,39}$. A ideia do tratamento até que o 
paciente atinja a remissão passa a ser questionada por alguns autores. $O$ problema dos sintomas "residuais" acaba acarretando em uso de doses maiores de medicações e, consequentemente, mais efeitos colaterais. Isto pode ser repensado através do conceito de temperamento ${ }^{40}$.

Poucos estudos abordam temperamentos afetivos e principalmente sua relação com o diagnóstico de transtornos do humor em todas as faixas etárias. Existem mais estudos relacionando temperamentos como fator de risco para suicídio e outros transtornos psiquiátricos. Em uma revisão sobre o assunto no PubMed com as palavras transtornos afetivos e transtornos do humor, chega-se ao número de 231 artigos. Porém, fazendo uma análise destes estudos verifica-se que apenas 21 apresentam relação com o diagnóstico de transtornos do humor (Figura 1).

A prevalência dos temperamentos afetivos depende do nível de corte ( $50 \%$ ou $75 \%$ ) da escala Memphis, Pisa, Paris e San Diego (TEMPSA). Um estudo usando o corte de $75 \%$ ou mais da escala TEMPS-A chegou ao resultado de que $40 \%$ da amostra em um serviço de transtornos do humor apresentavam critérios para ciclotimia. Outros $15 \%$ desta amostra teve 0 diagnóstico de hipertimia e $10 \%$ de distimia. Ao redor de $50 \%$ não apresentaram nenhum tipo de temperamento. Se o corte usado for de $50 \%$ dos itens da escala, a maioria dos sujeitos com transtornos do humor apresentariam as definições para temperamentos afetivos ${ }^{10}$.

Segundo a literatura, este seria o primeiro estudo a quantificar e a associar os temperamentos afetivos aos diagnósticos de transtornos do 


\section{humor em uma amostra de idosos brasileiros em ambulatório acadêmico de}

nível terciário.

Figura 1: Fluxograma da busca de artigos da revisão sistemática

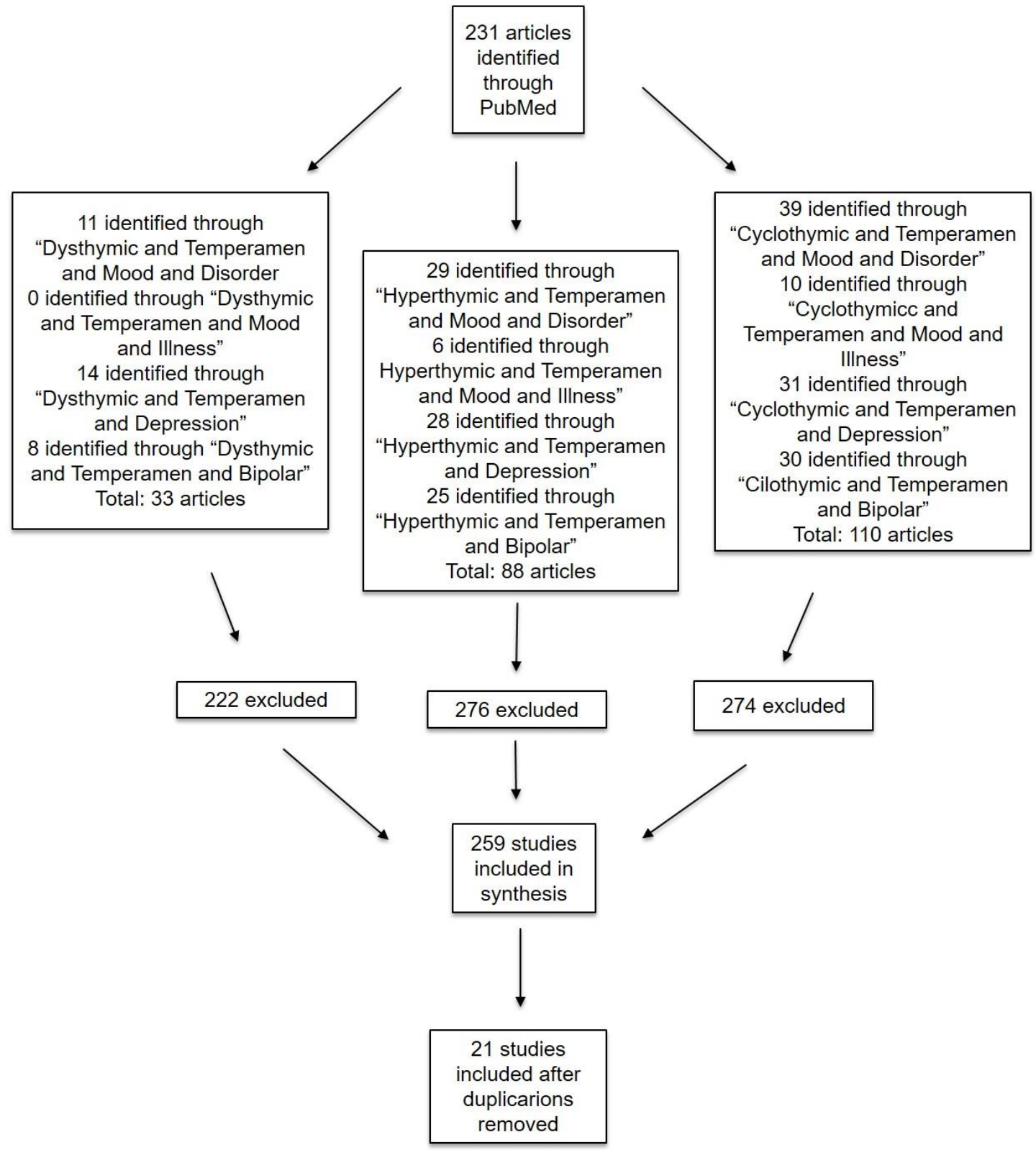




\section{OBJETIVOS}

\subsection{Objetivo Geral}

Avaliar os três principais tipos de temperamentos afetivos, por meio de escala validada, em idosos ambulatoriais com transtorno afetivo bipolar ou depressão maior, e em controles sem diagnósticos psiquiátricos de acordo com o DSM.

\subsection{Objetivos Específicos}

O primeiro objetivo é identificar e comparar a frequência dos três principais tipos de temperamentos afetivos (distimia, hipertimia e ciclotimia) entre idosos com os diagnósticos de transtorno afetivo bipolar ou de depressão maior, e em pacientes controles, ou seja, sem nenhum diagnóstico psiquiátrico. O segundo objetivo específico é identificar a associação entre os diagnósticos de transtorno afetivo bipolar e depressão maior e os três principais temperamentos afetivos, usando o corte de $75 \%$ da TEMPS-A. O terceiro objetivo é avaliar e comparar a frequência e a associação entre tentativa de suicídio, abuso infantil, abuso sexual e automutilação (desfechos secundários) e os três principais tipos de temperamentos afetivos em pacientes com doença maníaco depressiva. 


\section{HIPÓTESES}

Nossa principal hipótese neste projeto é a de que sujeitos ciclotímicos e distímicos seriam mais prevalentes no grupo de pacientes com diagnósticos psiquiátricos prévios (segundo DSM-5), enquanto que sujeitos com temperamento hipertímico seriam mais prevalentes entre o grupo que não apresenta diagnóstico psiquiátrico anterior. 


\section{JUSTIFICATIVA}

O curso das doenças psiquiátricas é frequentemente deixado em segundo plano dentro do sistema empregado pelo DSM-5, onde a avalição dos sintomas é central. Entender a relação entre os tipos de temperamento afetivo e os diagnósticos baseados pelo DSM-5 pode ajudar a esclarecer dificuldades e imprecisões psicopatológicas.

Para esse entendimento, a população idosa apresenta grande vantagem para o estudo destes conceitos, tendo em vista que apresenta o curso longitudinal quase completo das doenças mentais. Ademais, como estudos sobre temperamentos afetivos não são tão frequentes na literatura científica, principalmente em relação ao idoso, este estudo visa contribuir de forma relevante suprindo uma lacuna existente nesta linha de pesquisa. 


\section{MÉTODOS}

\subsection{Amostra}

O estudo avaliou 72 casos e 100 controles. O grupo denominado Caso foram divididos entre o diagnóstico de transtorno afetivo bipolar de qualquer tipo (TAB) e o de depressão maior. Os pacientes do grupo Caso foram diagnosticados pelos seus médicos assistentes. Os sujeitos são, em sua maioria, pacientes do Ambulatório de Geriatria da Faculdade de Medicina de Jundiaí, do Ambulatório de Alterações Comportamentais em Idosos do HCFMUSP (ACID) e do Ambulatório de especialidades médicas Dra. Jandira Mansur. Os 100 pacientes do grupo Controle, ou seja, pacientes sem nenhum diagnóstico psiquiátrico, foram provenientes do Ambulatório de Reumatologia do Hospital Evangélico de Curitiba. Apesar destes últimos virem de um serviço de reumatologia, se verificou atentamente a ausência de diagnóstico psiquiátrico e o seguimento temporal acima de 12 meses.

Os critérios de inclusão foram os seguintes: pacientes idosos, com idade igual ou maior a 65 anos; com diagnósticos prévios de TAB ou depressão maior; em acompanhamento clínico regular; compensados de suas doenças clínicas; sem cirurgias nos últimos 3 meses; colaborativos com os testes realizados no protocolo de investigação. O grupo Controle foi formado pelos mesmos critérios, mas com a diferença de não apresentarem diagnóstico de TAB ou depressão. 
Os pacientes que apresentaram qualquer síndrome demencial, comprometimento funcional ou sensorial que os impediram de se submeter a aplicação dos testes propostos, além de acidente vascular cerebral nos últimos 6 meses, estar em tratamento oncológico ou recusa em assinar o Termo de Consentimento Livre e Esclarecido (TCLE), foram excluídos da amostra (Figura 2).

Figura 2: Fluxograma de inclusão de pacientes

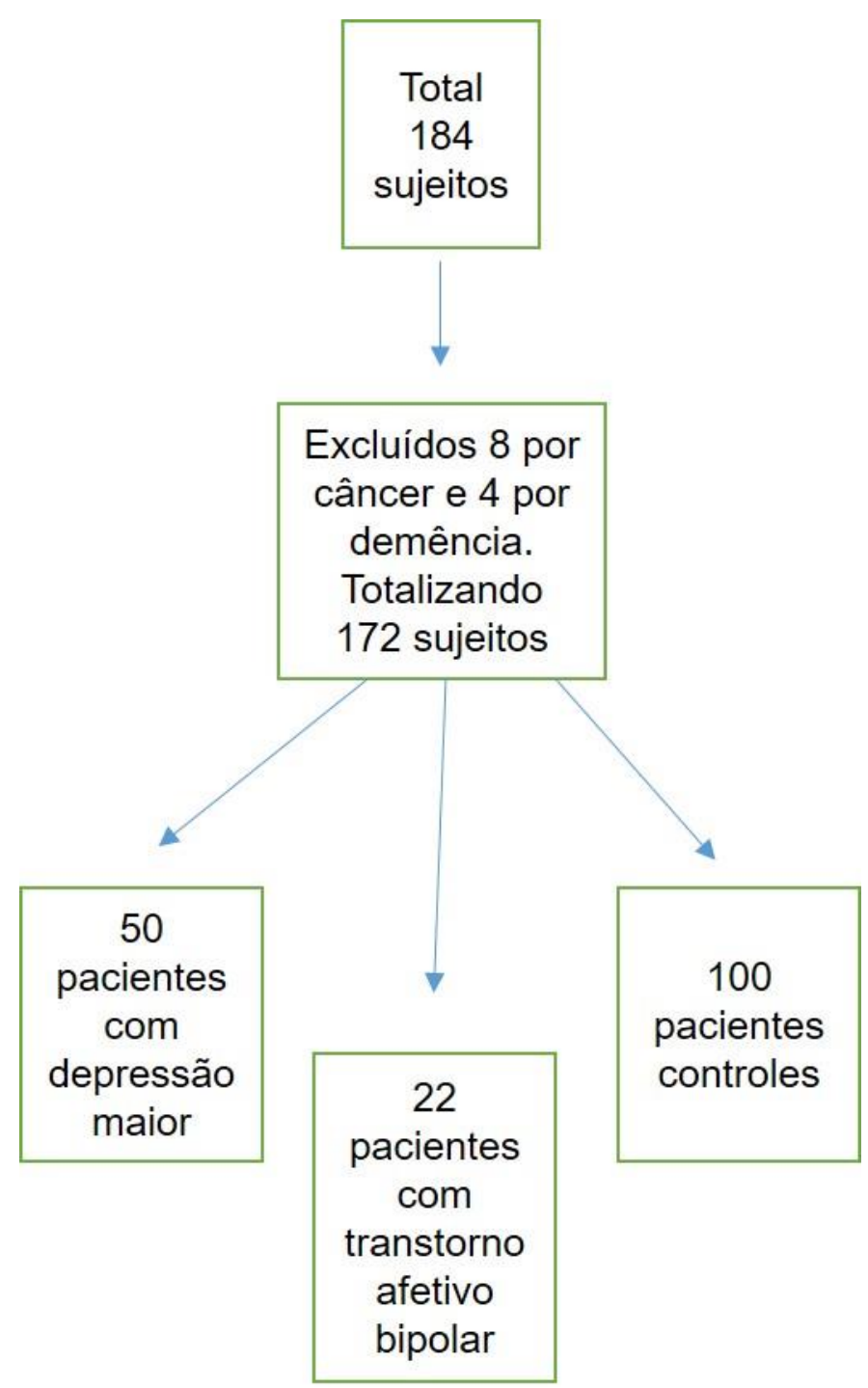




\subsection{Instrumentos de Avaliação Clínica}

Todos os pacientes foram submetidos ao Mini Exame do Estado Mental na versão brasileira para avaliação cognitiva. Foram aceitos pacientes com pontuação acima de 18 pontos, de acordo com a escolaridade da amostra e respeitando critérios publicados anteriormente e de ampla aceitação ${ }^{41,42}$. Foram usadas para avaliação dos sintomas de depressão, ansiedade e mania as escalas HAMD 17 itens para depressão, a escala de Hamilton para ansiedade e a escala YMRS para avaliação de $\operatorname{mania}^{43,44,45}$.

Os temperamentos afetivos foram avaliados através da escala/auto questionário TEMPS-A (Temperament Evaluation Memphis, Pisa, Paris and San Diego) Rio de Janeiro ${ }^{46}$. O questionário TEMPS-A já foi traduzido para mais de 25 línguas. Na versão brasileira este questionário é formado por 6 subescalas, cada uma formada por questões com respostas verdadeiro e falso, sendo um total de 45 perguntas. Cada subescala (não claramente explícita na entrevista) corresponde a um temperamento. Nesta versão foram utilizados os temperamentos distímicos, ciclotímicos, hipertímicos, irritável e preocupado. Este trabalho focou-se apenas nos três principais tipos de temperamento, ou seja, distímico, ciclotímico e hipertímico. Os temperamentos ansioso e preocupado só foram adicionados nas versões mais recentes da escala americana ${ }^{28}$. A pontuação na versão brasileira deve-se contar "1" para respostas verdadeiras e "0" para respostas erradas, multiplicando-se o resultado de cada subescala por 12.5. Pode-se ter um 
resultado entre $0-100$, pois as subescalas de ciclotimia, distimia e hipertimia são formadas por 8 itens cada. Este estudo optou por usar a escala TEMPSA como uma variável dicotômica com corte de $75 \%$ das respostas afirmativas para determinar o temperamento dominante, seguindo a metodologia de um recente estudo ${ }^{10}$. Ainda se fazem estudos de exploração diagnóstica deste instrumento para sua utilização na prática clínica ${ }^{47}$. É importante entender que os temperamentos não são auto-excludentes, ou seja, os pacientes podem marcar positivo para mais de um tipo. Quando se usa o corte de $75 \%$ esta sobreposição diminui.

\subsection{Consentimento Informado}

Todos os procedimentos clínicos de interesse da pesquisa foram informados aos sujeitos, e quando necessário ao seu acompanhante, sendo então obtida a assinatura do Termo de Consentimento Livre e Esclarecido, devidamente aprovado, assim como este estudo, pelo Comitê de Ética do Hospital das Clínicas da Faculdade de Medicina da Universidade de São Paulo e pelo Departamento de Psiquiatria da mesma Faculdade.

\subsection{Análise Estatística}

A análise estatística foi realizada através do programa " $R$ " versão 3.5.148. Foi incluída na análise estatística a descrição da amostra segundo idade, escolaridade, estado civil, religião e gênero. Os antecedentes psiquiátricos como internação psiquiátrica pregressa, eletroconvulsoterapia, tentativas de suicídio, automutilação e abuso na infância foram avaliados. 
Os antecedentes psiquiátricos familiares foram descritos, assim como comorbidades clínicas. A amostra foi estratificada de acordo com os diagnósticos de depressão e TAB, além dos sujeitos controles que não apresentavam nenhum dos dois diagnósticos. As variáveis continuas são mostradas na tabela segundo distribuição de média e desvio-padrão. As variáveis categóricas estão demonstradas segundo a sua porcentagem. Nas tabelas não foram incluídos o p valor, pois em estudos epidemiológicos, como caso-controle, não há a hipótese de que os grupos sejam similares ${ }^{49}$.

Com relação a análise de medida de associação entre os diagnósticos de TAB e depressão e os três temperamentos, além de desfechos secundários (como tentativas de suicídio, abuso na infância, abuso sexual e automutilação) foi usado o risco relativo (RR), pois não havia poder estatístico para se montar modelos de regressão logística, o que seria o ideal para se ajustar em relação a possíveis confundidores. Muitos autores discutem o uso do risco relativo ao invés do uso da razão de probabilidade, ou em inglês odds ratio, em estudos caso-controle. O risco relativo se aproxima da razão de probabilidade e é muito mais intuitivo. A razão de probabilidade ou odds ratio, deve ser usado, matematicamente, como resultado de regressões logísticas ${ }^{50}$. 


\section{RESULTADOS}

Foram avaliados 22 pacientes com diagnóstico de TAB, 50 pacientes com diagnóstico de depressão maior e 100 sujeitos do grupo controle. As características sóciodemográficas encontram-se na Tabela 1, junto com os antecedentes psiquiátricos da amostra. Os pacientes do grupo com TAB apresentaram mais internações que o grupo controle, além de mais tentativas de suicídio e história de automutilação. Abuso sexual e outros tipos de abuso na infância também foram mais frequentes no grupo com diagnóstico de TAB. Os antecedentes psiquiátricos familiares encontram-se na Tabela 2. Os pacientes com TAB apresentaram maior frequência de familiares com história de TAB, depressão, esquizofrenia e abuso de drogas. Na Tabela 3, apresenta-se as comorbidades clínicas. O grupo com TAB apresentou maior incidência de diabetes, hipotireoidismo, infarto do miocárdio e dor crônica, quando comparados ao grupo controle.

Os resultados das escalas diagnósticas utilizadas são observadas na Tabela 4. Em comparação ao grupo controle, os pacientes com TAB apresentaram mais sintomas de ansiedade. Com relação a incidência de tipos de temperamentos, os pacientes com TAB apresentaram uma porcentagem maior de distimia $(18,2 \%$ comparados aos $5 \%$ do grupo controle). No entanto, o tipo de temperamento mais comum neste grupo foi o hipertímico. A incidência de temperamento hipertímico foi de $77 \%$ no grupo controle. Todos os pacientes neste grupo apresentaram algum tipo de temperamento. 
Os pacientes com depressão maior apresentaram mais internações psiquiátricas, tentativas de suicídio e tratamento psicoterápico. Com relação aos antecedentes psiquiátricos familiares, os pacientes do grupo com depressão apresentaram mais história de depressão, suicídio e demência, quando comparados ao grupo controle. Este grupo apresentou mais diabetes, obesidade e dor crônica como comorbidades clínicas. Os pacientes com depressão apresentaram mais prevalência de temperamentos hipertímico e ciclotímico. Destes pacientes, 24\% não apresentaram nenhum tipo de temperamento. Da amostra inteira, 17,4\% não apresentaram nenhum temperamento.

Tabela 1: Características sociodemográficas e antecedentes psiquiátricos da amostra

\begin{tabular}{|c|c|c|c|c|}
\hline & TAB $(n=22)$ & $\begin{array}{l}\text { Depressão } \\
(\mathrm{n}=50)\end{array}$ & $\begin{array}{l}\text { Controles } \\
(n=100)\end{array}$ & $\begin{array}{l}\text { Total } \\
(\mathrm{N}=172)\end{array}$ \\
\hline Idade(media \pm DP) & $70,9 \pm 5,3$ & $74,2 \pm 6,4$ & $72,3 \pm 5,3$ & $72,6 \pm 5,7$ \\
\hline Escolaridade(media $\pm \mathrm{DP})$ & $6,3 \pm 5,7$ & $5,5 \pm 3,8$ & $4,5 \pm 3,3$ & $5,0 \pm 3,9$ \\
\hline Estado civil-viúvo (\%) & 27,2 & 46,0 & 41,0 & 41,0 \\
\hline Aposentado (\%) & 86,3 & 82,0 & 90,0 & 87,2 \\
\hline Religião (\%) -Católica & 59,1 & 70,0 & 75,0 & 70,9 \\
\hline Gênero-Feminino (\%) & 77,2 & 86,0 & 88,0 & 86,0 \\
\hline Cor - Branco (\%) & 72,7 & 94,0 & 92,0 & 90,1 \\
\hline $\begin{array}{l}\text { Internação psiquiátrica- } \\
\text { sim (\%) }\end{array}$ & 50 & 8,0 & 0 & 8,7 \\
\hline $\begin{array}{l}\text { Eletroconvulsoterapia- } \\
\text { Sim (\%) }\end{array}$ & 18,1 & 2,0 & 0 & 2,9 \\
\hline Psicoterapia- sim (\%) & 50,0 & 32,0 & 9,0 & 20,9 \\
\hline $\begin{array}{l}\text { Tentativa de suicídio-sim } \\
(\%)\end{array}$ & 22,7 & 10,0 & 1,0 & 6,4 \\
\hline $\begin{array}{l}\text { Abuso na infância-sim- } \\
(\%)\end{array}$ & 27,2 & 6,0 & 2,0 & 6,4 \\
\hline Automutilação-sim- (\%) & 13,6 & 0 & 0 & 1,7 \\
\hline Abuso sexual -sim - (\%) & 18,2 & 4,0 & 3,0 & 5,2 \\
\hline
\end{tabular}

$\mathrm{DP}=$ Desvio Padrão TAB=Transtorno Afetivo Bipolar 
Tabela 2: Antecedentes psiquiátricos familiares

\begin{tabular}{|l|l|l|l|l|}
\hline & TAB(n=22) & Depressão(n=50) & Controles $(\mathrm{n}=100)$ & Total(N=172) \\
\hline $\begin{array}{l}\text { Depressão } \\
(\%)\end{array}$ & 45,4 & 38,0 & 27,0 & 32,5 \\
\hline $\begin{array}{l}\text { Ansiedade } \\
(\%)\end{array}$ & 18,2 & 6,0 & 6,0 & 7,5 \\
\hline $\begin{array}{l}\text { Transtorno } \\
\text { afetivo } \\
\text { bipolar (\%) }\end{array}$ & 36,4 & 8,0 & 3,0 & 8,7 \\
\hline Suicídio (\%) & 13,6 & 16,0 & 8,0 & 11,0 \\
\hline $\begin{array}{l}\text { Esquizofrenia } \\
\text { (\%) }\end{array}$ & 18,2 & 6,0 & 0 & 4,1 \\
\hline $\begin{array}{l}\text { Abuso de } \\
\text { álcool (\%) }\end{array}$ & 36,4 & 36,0 & 42,0 & 39,5 \\
\hline $\begin{array}{l}\text { Abuso de } \\
\text { drogas (\%) }\end{array}$ & 27,3 & 10,0 & 10,0 & 12,2 \\
\hline $\begin{array}{l}\text { Demência } \\
\text { (\%) }\end{array}$ & 9,1 & 10,0 & 2,0 & 5,2 \\
\hline $\begin{array}{l}\text { Sem história } \\
\text { familiar (\%) }\end{array}$ & 18,2 & 28,0 & 36,0 & 31,4 \\
\hline
\end{tabular}

$\mathrm{TAB}=$ Transtorno Afetivo Bipolar 
Tabela 3: Comorbidades clínicas

\begin{tabular}{|c|c|c|c|c|}
\hline & $\begin{array}{l}\text { TAB } \\
(n=22)\end{array}$ & Depressão(n=50) & Controles $(\mathrm{n}=100)$ & Total $(\mathrm{N}=172)$ \\
\hline Diabetes (\%) & 27,3 & 24,0 & 17,0 & 20,3 \\
\hline $\begin{array}{l}\text { Hipertensão } \\
\text { arterial (\%) }\end{array}$ & 50 & 72,0 & 60,0 & 62.2 \\
\hline $\begin{array}{l}\text { Acidente } \\
\text { vascular } \\
\text { cerebral (\%) }\end{array}$ & 0 & 6,0 & 6,0 & 5,2 \\
\hline $\begin{array}{l}\text { Infarto do } \\
\text { miocárdio (\%) }\end{array}$ & 4,5 & 2,0 & 4,0 & 3,5 \\
\hline $\begin{array}{l}\text { Insuficiência } \\
\text { coronariana } \\
(\%)\end{array}$ & 0 & 4,0 & 2,0 & 2,3 \\
\hline $\begin{array}{l}\text { Insuficiência } \\
\text { cardíaca } \\
\text { congestiva (\%) }\end{array}$ & 0 & 2,0 & 2,0 & 1,7 \\
\hline $\begin{array}{l}\text { Insuficiência } \\
\text { renal crônica } \\
(\%)\end{array}$ & 4,5 & 2,0 & 0 & 1,2 \\
\hline $\begin{array}{l}\text { Doença } \\
\text { pulmonar } \\
\text { obstrutiva } \\
\text { crônica (\%) }\end{array}$ & 0 & 2,0 & 1,0 & 1,2 \\
\hline Obesidade (\%) & 4,5 & 4,0 & 2,0 & 2,9 \\
\hline Dor crônica (\%) & 4,5 & 4,0 & 3,0 & 3,5 \\
\hline $\begin{array}{l}\text { Hipotireoidismo } \\
(\%)\end{array}$ & 18,2 & 22,0 & 23,0 & 22,1 \\
\hline $\begin{array}{l}\text { Artrite/ artrose } \\
(\%)\end{array}$ & 0 & 38,0 & 65,0 & 48,8 \\
\hline $\begin{array}{l}\text { Osteoporose } \\
(\%)\end{array}$ & 4,5 & 14,0 & 32,0 & 23,2 \\
\hline $\begin{array}{l}\text { Sem } \\
\text { comorbidades } \\
\text { clínica. }\end{array}$ & 36,4 & 4,0 & 0 & 7,0 \\
\hline
\end{tabular}

TAB=Transtorno Afetivo Bipolar 
Tabela 4: Escalas diagnósticas

\begin{tabular}{|c|c|c|c|c|}
\hline & $\mathrm{TAB}(\mathrm{n}=22)$ & Depressão( $(\mathrm{n}=50)$ & Controles $(n=100)$ & $\begin{array}{l}\text { Total } \\
(\mathrm{N}=172)\end{array}$ \\
\hline $\begin{array}{l}\text { Escala de } \\
\text { Hamilton } \\
\text { para } \\
\text { ansiedade } \\
\text { (media } \pm D P)\end{array}$ & $11,4 \pm 10,6$ & $9,4 \pm 7,6$ & $4,7 \pm 3,8$ & $6,9 \pm 6,8$ \\
\hline $\begin{array}{l}\text { Mini exame } \\
\text { do estado } \\
\text { mental } \\
(\text { media } \pm D P)\end{array}$ & $25,8 \pm 4,0$ & $26,3 \pm 3,1$ & $26,7 \pm 3,1$ & $26,5 \pm 3,2$ \\
\hline $\begin{array}{l}\text { TEMPS-A } \\
\text { Distimia } \\
75(\%)\end{array}$ & 18,2 & 14,0 & 5,0 & 9,3 \\
\hline $\begin{array}{l}\text { TEMPS-A } \\
\text { Ciclotimia } \\
75(\%)\end{array}$ & 50,0 & 38,0 & 12,0 & 24,4 \\
\hline $\begin{array}{l}\text { TEMPS-A } \\
\text { Hipertimia } \\
75(\%)\end{array}$ & 59,1 & 48,0 & 77,0 & 66,2 \\
\hline $\begin{array}{l}\text { TEMPS-A } \\
\text { nenhum } 75 \\
(\%)\end{array}$ & 0 & 24,0 & 18,0 & 17,4 \\
\hline $\begin{array}{ll}\text { Escala de } \\
\text { mania de } \\
\text { Young } \\
\text { (media } \pm D P)\end{array}$ & $2,3 \pm 6,2$ & $2,3 \pm 3,8$ & $3,2 \pm 2,8$ & $2,8 \pm 3,7$ \\
\hline $\begin{array}{l}\text { Escala de } \\
\text { Hamilton } 17 \\
\text { para } \\
\text { Depressão } \\
\text { (media } \pm D P \text { ) }\end{array}$ & $4,7 \pm 5,1$ & $4,5 \pm 5,3$ & $2,0 \pm 1,7$ & $3,1 \pm 3,8$ \\
\hline
\end{tabular}

DP=Desvio Padrão TAB=Transtorno Afetivo Bipolar 


\subsection{Medida de associação entre diagnósticos psiquiátricos (TAB e depressão) e temperamentos.}

\subsubsection{TAB e Temperamentos}

Dos sujeitos com diagnóstico de TAB, 18,2\% apresentaram critérios para temperamento distímico (contra $5 \%$ dos controles), resultando em risco relativo de 3,6 e com um intervalo de confiança (IC) de $95 \%$ de 1,1-12,4. Assim, verificou-se que pacientes com distimia tem um risco de quase quatro vezes maior de serem diagnosticados com TAB (Tabela 5).

Com relação ao temperamento ciclotímico, metade dos pacientes com TAB apresentaram critérios. Já entre os controles, apenas 12\% preencheram critérios para ciclotimia, resultando em um risco relativo de 4,2 (IC 95\% 2,1$8,2)$.

Nesta amostra, $59,1 \%$ dos pacientes com TAB apresentaram critérios para hipertimia, enquanto que $77 \%$ da amostra de controles fecharam critérios para este tipo de temperamento, resultando em um RR de 0,8 (IC 95\% 0,51,1). Isto representa que mesmo não sendo estatisticamente significante, os pacientes hipertímicos tem um possível risco 20\% menor de serem diagnosticados com TAB.

Tabela 5: Risco Relativo TAB

\begin{tabular}{|l|l|l|}
\hline Hipertimia & $\mathrm{RR}=0.8$ & $95 \% \mathrm{IC}$ 0.5-1.1 \\
\hline Ciclotimia & $\mathrm{RR}=4.2$ & $95 \%$ IC 2.1-8.2 \\
\hline Distimia & $\mathrm{RR}=3.6$ & $95 \% \mathrm{IC} 1.1-12.4$ \\
\hline
\end{tabular}




\subsubsection{Depressão e Temperamentos}

Da amostra de pacientes com diagnóstico de depressão maior, 14\% fecharam diagnóstico para distimia, enquanto que os controles apresentaram $5 \%$, resultando em um RR de 2,8 (IC 95\% 0,9-8,3). (Tabela 6)

Os pacientes ciclotímicos com diagnóstico de depressão representam $38 \%$ da amostra. Já entre os controles, pacientes ciclotímicos representam 12\%, resultando em um RR de 3,2 (IC 95\% 1,7-6,0).

Pacientes deprimidos com diagnóstico de temperamento hipertímico representam $48 \%$. Entre os controles essa parcela aumentou para $77 \%$, resultando em um RR de 0,6 (IC 95\% 0,4-0,8). Pacientes hipertímicos apresentaram um risco $40 \%$ menor de apresentarem o diagnóstico de depressão maior.

Tabela 6: Risco Relativo Depressão

\begin{tabular}{|l|l|l|}
\hline Hipertimia & $\mathrm{RR}=0.6$ & $95 \% \mathrm{IC}$ 0.4-0.8 \\
\hline Ciclotimia & $\mathrm{RR}=3.2$ & $95 \%$ IC 1.7-6.0 \\
\hline Distimia & $\mathrm{RR}=2.8$ & $95 \% \mathrm{IC}$ 0.9-8.3 \\
\hline
\end{tabular}

RR=Risco Relativo

\subsubsection{Desfechos secundários}

Usando-se o conceito de doença maníaco depressiva, ou seja, pacientes com TAB e depressão maior, analisou-se a amostra de pacientes com este diagnóstico entendendo a relação dos três tipos de temperamento e tentativas de suicídio, abuso na infância, abuso sexual e automutilação (desfechos secundários). 
Com relação a tentativa de suicídio, $36,3 \%$ dos pacientes distímicos, $16,6 \%$ dos pacientes ciclotímicos e $8,1 \%$ dos pacientes hipertímicos fizeram tentativas de suicídio. Já entre os pacientes controles, apenas 1\% relataram tentativas de suicídio. Isto corresponde respectivamente aos seguintes RR: 36,3 (95\% IC 4,4-297,2), 16,7 (95\% IC 2,0-137,2) e 8,0 (95\%IC 0,9-75,5).

Com relação ao abuso na infância, 18,1\% dos pacientes distímicos (RR 9,1, 95\% IC 1,4-58,3) relataram serem vitimas de abuso. Entre os pacientes ciclotímicos $22,5 \%$ (RR $11,7,95 \%$ IC 2,5-53,2) e entre os pacientes hipertímicos 8,1\% (RR 4,1, 95\% IC 0,7-23,7) sofreram abuso na infância. Pacientes controles apresentaram apenas 2\% de abuso na infância.

Dos pacientes distímicos, 18,1\% sofreram abuso sexual (RR 6,1, 95\% IC 1,1-32,4). Já entre os ciclotímicos, $13,3 \%$ dos pacientes relataram abuso sexual (RR 4,4, 95\% IC1,1-18,7). Entre os hipertímicos, 5,4\% dos pacientes relataram serem vítimas de abuso sexual (RR 1,8, 95\% IC 0,3-10,3). Contudo, no grupo controle $3 \%$ dos pacientes sofreram abuso sexual em algum período da vida.

Pacientes com comportamento de automutilação representam 9,1\% dos pacientes distímicos, $6,6 \%$ dos ciclotímicos e 2,7\% dos hipertímicos. Já entre os controles nenhum paciente apresentou este tipo de comportamento. Os riscos relativos são os seguintes: distímicos 25,2 (95\% IC 1,1-585,8), ciclotímicos 16,2 (95\% IC 0,8-330,3) e hipertímicos 8,0 (95\% IC 0,3-191,5). 


\section{DISCUSSÃO}

Segundo nosso conhecimento, este é o primeiro estudo que associa temperamentos afetivos a transtornos do humor em idosos. Alguns poucos estudos foram feitos em outras faixas etárias ${ }^{51,52}$. A grande maioria destes estudos relacionaram os temperamentos afetivos aos diversos tipos de transtorno bipolar. Neste estudo, a grande maioria dos participantes apresentaram algum tipo de temperamento. Diferente de outros estudos, nesta parcela da população a maioria dos pacientes apresentaram temperamentos do tipo hipertímico, seguido do temperamento ciclotímico (66,2\% e $24,4 \%$, respectivamente). Um estudo avaliou pacientes com média de 38 anos de idade e os temperamentos mais prevalentes foram o ciclotímico e o hipertímico, quando utilizado o corte de $75 \%$ (40\% e $30 \%$, respectivamente $)^{10}$. Sendo os pacientes hipertímicos mais prevalentes neste estudo, qual seria a relação entre este tipo de temperamento e a longevidade? Poucos estudos relacionam estas duas variáveis. Um deles estudou a personalidade de pacientes com idade média de 50 anos, através de outras escalas (uma delas a NEO-PI) e concluiu que pacientes com características parecidas com pacientes hipertímicos eram mais longevos. Característica como extroversão diminuiu a mortalidade em $13 \%$, enquanto baixo neuroticismo diminuiu-a em $15 \%{ }^{53}$. Outro estudo mostrou que os pacientes hipertímicos tiveram uma melhor funcionalidade após o tratamento para mania, quando comparados aos pacientes ciclotímicos que apresentaram sintomas depressivos residuais ${ }^{51}$. Novos estudos utilizando melhores metodologias são necessários. 
Previamente, pacientes participantes de um estudo realizado na Itália, com diagnóstico de depressão, apresentaram temperamento afetivo em 12,3\%, distímico em 7,5\% e hipertímico em 2,8\%52. Em um outro estudo japonês, que relacionou os diagnósticos de TAB e depressão maior, todos os 46 pacientes apresentaram algum tipo de temperamento. Entre os pacientes com TAB, $70 \%$ apresentaram temperamento ciclotimico, 30\% apresentaram temperamento hipertímico e $40 \%$ distímico. Entre os pacientes com depressão maior, $72 \%$ apresentaram temperamento ciclotímico, $31 \%$ hipertímico e $75 \%$ distímico 54 .

$\mathrm{Na}$ amostra do nosso estudo, pacientes com diagnóstico de depressão apresentaram temperamento hipertímico em $48 \%$, ciclotímico em $38 \%$ e distímico em $14 \%$. No entanto, a associação mais forte foi com temperamento ciclotímico, aumentando em mais de três vezes a chance de se ter o diagnóstico de depressão. Os pacientes com diagnóstico de temperamento hipertímico apresentaram uma chance diminuída de ter diagnostico de depressão maior em 40\%. Com relação ao TAB, nosso estudo detectou 18,2\% de temperamento distímico, $50 \%$ de pacientes ciclotímicos e 59,1\% de hipertímicos. A maior associação foi também com o temperamento ciclotímico. Um outro estudo relacionou o temperamento afetivo ao uso de antidepressivos e concluiu que os temperamentos hipertímicos e ciclotímicos estão mais associados a quadros de mania ${ }^{55}$. Os pacientes com depressão unipolar e temperamento ciclotímico responderiam melhor a estabilizadores de humor? 
Nos últimos anos tem-se retomado os conceitos Kraepelinianos em relação a doença maníaco depressiva, através do conceito de espectro bipolar ${ }^{9}$. Para tanto, em uma das análises usamos uma amostra com pacientes com TAB e depressão e analisamos os efeitos dos temperamentos em relação a suicídio, abuso na infância, abuso sexual e automutilação. Ao nosso conhecimento este também é o primeiro estudo em que se aplica uma análise como está em idosos.

Pacientes distímicos apresentaram uma chance aumentada com relação ao suicídio ( $R R=36,3,95 \%$ IC 4,4-297,2). Já pacientes hipertímicos não apresentaram uma relação estatisticamente significante ( $R R=8,0,95 \%$ IC 0,9-75,5), sendo que clinicamente pode haver um efeito protetivo, de acordo com este intervalo de confiança. Estes resultados estão alinhados com dois outros estudos que demonstram que o temperamento distímico pode ser fator de risco para suicídio. Importante salientar que em um destes estudos comprovou-se que houve um aumento de mais de nove vezes no risco de suicídio, quando comparado a pessoas sem o diagnostico de doença mental. Já o temperamento hipertímico foi considerando neutro ou protetivo nestes estudos (OR=0,9, 95\% IC 0,3-2,5) 56,57 .

Estudos mostram que os temperamentos distímicos e ciclotímicos estão mais associados a abuso na infância ${ }^{58,59}$. Em nosso estudo, pacientes com distimia e ciclotimia apresentaram um risco relativo muito parecido, quando comparados ao grupo controle. Distímicos tiveram um risco nove vezes maior, e ciclotímicos onze vezes maior de sofrer abuso na infância. Já 
os hipertímicos apresentaram um risco quatro vezes maior, mas não estatisticamente significante.

Com relação a abuso sexual e sua associação com temperamentos, nenhum estudo prévio foi encontrado para fins comparativos. Nosso estudo apresenta uma associação, novamente, mais forte para distimia $(R R=6,1$, $95 \%$ IC 1,1-32,4) e ciclotimia (RR=4,4, 95\% IC 1,1-18,7).

Alguns autores entendem que no caso de abuso, podendo ser de qualquer tipo, o temperamento distímico ou ciclotímico seria um mediador, aumentado as chances de piores desfechos para os pacientes ${ }^{60}$. Já os pacientes hipertímicos amenizariam os piores desfechos do trauma, pois melhoram a resiliência. Este seria um dos motivos pelos quais nem todos os pacientes que sofrem traumas desenvolvem transtorno de estresse pós traumático61.

Ao menos dois estudos associam a automutilação ao temperamento ciclotímico. Este dado diverge do nosso no qual a maior associação significativa foi com o temperamento distímico ( $R R=25,2,95 \%$ IC 1,1-585,8). Esta discrepância pode ser causada pela idade da amostra, sendo que os dois estudos em questão tem idades medias menores que este estudo ${ }^{62,63}$.

Este estudo apresenta algumas limitações que merecem destaque. A amostra foi conveniente dentro de serviços universitários, os diagnósticos de TAB ou depressão foram feitos por psiquiatras sem o uso de nenhuma entrevista validada e estruturada. A ideia de não usar nenhuma entrevista estruturada foi com o intuito de entender o papel dos temperamentos dentro 
de um desenho de estudo de "vida real" (real-world evidence). A generalização deste estudo fica comprometida, pois mais de $90 \%$ da amostra é formada por caucasianos e $86 \%$ são mulheres. Outra limitação que este estudo apresenta é a baixa escolaridade da amostra (média de 5 anos de frequência escolar), o que dificulta o entendimento das escalas diagnósticas e, portanto, uma dificuldade na aplicação do principal método de avaliação do estudo.

A conclusão é de que sujeitos com temperamento ciclotímico tem um risco maior de serem diagnosticados tanto com TAB quanto com depressão, quando comparados aos controles. Entretanto, pacientes com temperamento hipertímico possuem um risco diminuído de apresentarem diagnóstico de depressão.

Pacientes distímicos apresentaram mais chance de cometerem tentativas de suicídio, automutilação e serem vitimas de abuso sexual. Já pacientes ciclotímicos apresentam mais risco de sofrerem abuso na infância.

Futuros estudos com desenhos de pesquisa diferentes deste estudo transversal caso-controle serão necessários para analisar o papel dos temperamentos em relação aos diagnósticos de transtornos do humor. Além disto, são importantes a realização de estudos de segmentos para entender o papel dos temperamentos nos episódios de recidiva dos transtornos do humor. 


\section{REFERÊNCIAS BIBLIOGRÁFICAS}

1. Ghaemi SN. The concepts of psychiatry: a pluralistic approach to the mind and mental illness. Baltimore: Johns Hopkins University Press; 2007.

2. Marneros A. Psychiatry's 200th birthday. Br J Psychiatry. 2008;193:13.

3. Binder DK, Schaller K, Clusmann $\mathrm{H}$. The seminal contributions of Johann-Christian Reil to anatomy, physiology, and psychiatry. Neurosurgery. 2007;61:1091-6.

4. Graham G. The disordered mind: an introduction to philosophy of mind and mental illness. 2nd ed. New York: Routledge; 2013.

5. Alexander F, Selesnick S. The history of psychiatry: an evaluation of psychiatric thought and practice from prehistoric times to the present. New York: Harper \& Row; 1966.

6. Akiskal HS. Dysthymia and cyclothymia in psychiatric practice a century after Kraepelin. J Affect Disord. 2001;62:17-31.

7. Ghaemi SN. Bipolar spectrum: a review of the concept and a vision for the future. Psychiatry Investig. 2013;10:218-24.

8. Ghaemi SN. On depression: drugs, diagnosis, and despair in the modern world. Baltimore: Johns Hopkins University Press; 2013.

9. Ghaemi SN, Dalley S. The bipolar spectrum: conceptions and misconceptions. Aust N Z J Psychiatry. 2014;48:314-24.

10. Vöhringer PA, Whitham EA, Thommi SB, Holtzman NS, Khrad H, Ghaemi SN. Affective temperaments in clinical practice: a validation study in mood disorders. J Affect Disord. 2012;136:577-80.

11. Koukopoulos A, Tundo A. A mixed depressive syndrome. Clin Neuropharmacol. 1992;15 Suppl 1:626A-7A.

12. Byers AL, Yaffe K, Covinsky KE, Friedman MB, Bruce ML. High occurrence of mood and anxiety disorders among older adults: The National Comorbidity Survey Replication. Arch Gen Psychiatry. 2010;67:489-96.

13. Reynolds K, Pietrzak RH, El-Gabalawy R, Mackenzie CS, Sareen J. Prevalence of psychiatric disorders in U.S. older adults: findings from a nationally representative survey. World Psychiatry. 2015;14:74-81.

14. Ritter PL, Dal Pai D, Belmonte-de-Abreu P, Camozzato A. Trends in 
elderly psychiatric admissions to the Brazilian public health care system. Rev Bras Psiquiatr. 2016;38:314-7.

15. Robins E, Guze SB. Establishment of diagnostic validity in psychiatric illness: its application to schizophrenia. Am J Psychiatry. 1970;126:983-7.

16. Ghaemi SN. Taking disease seriously in DSM. World Psychiatry. 2013;12:210-2.

17. Ghaemi SN. Toward a Hippocratic psychopharmacology. Can J Psychiatry. 2008;53:189-96.

18. Thomas J, Grey I. From Black Bile to the Bipolar Spectrum: A Historical Review of the Bipolar Affective Disorder Concept. Arch Depress Anxiety. 2016;2:010-5.

19. Roccatagliata G. A history of ancient psychiatry. New York: Praeger; 1986.

20. Rihmer Z, Akiskal KK, Rihmer A, Akiskal HS. Current research on affective temperaments. Curr Opin Psychiatry. 2010;23:12-8.

21. Kretschmer E. Physique and character: an investigation of the nature of constitution and of the theory of temperament. London: Kegan Paul, Trench,Trubner; 1925.

22. Brieger $P$, Marneros $A$. Dysthymia and cyclothymia: historical origins and contemporary development. J Affect Disord. 1997;45:117-26.

23. Nigg JT. Temperament and developmental psychopathology. J Child Psychol Psychiatry. 2006;47:395-422.

24. Akiskal HS, Hirschfeld RM, Yerevanian BI. The relationship of personality to affective disorders. Arch Gen Psychiatry. 1983;40:80110.

25. Chodoff P. The depressive personality. A critical review. Arch Gen Psychiatry. 1972;27:666-73.

26. Klein DN. Depressive personality: reliability, validity, and relation to dysthymia. J Abnorm Psychol. 1990;99:412-21.

27. Akiskal HS, Bourgeois ML, Angst J, Post R, Moller HJ, Hirschfeld R. Re-evaluating the prevalence of and diagnostic composition within the broad clinical spectrum of bipolar disorders. J Affect Disord. 2000;59 Suppl. 1:S5-S30.

28. Akiskal HS, Akiskal KK, Haykal RF, Manning JS, Connor PD. TEMPSA: Progress towards validation of a self-rated clinical version of the 
Temperament Evaluation of the Memphis, Pisa, Paris, and San Diego Autoquestionnaire. J Affect Disord. 2005;85:3-16.

29. Kwapil TR, DeGeorge D, Walsh MA, Burgin CJ, Silvia PJ, BarrantesVidal N. Affective temperaments: unique constructs or dimensions of normal personality by another name? J Affect Disord. 2013;151:88290.

30. Evans LM, Akiskal HS, Greenwood TA, Nievergelt CM, Keck PE Jr, McElroy SL, Sadovnick AD, Remick RA, Schork NJ, Kelsoe JR. Suggestive linkage of a chromosomal locus on 18p11 to cyclothymic temperament in bipolar disorder families. Am J Med Genet B Neuropsychiatr Genet. 2008;147:326-32.

31. Lewinsohn PM, Klein DN, Durbin EC, Seeley JR, Rohde P. Family study of subthreshold depressive symptoms: risk factor for MDD? J Affect Disord. 2003;77:149-57.

32. Chiaroni P, Hantouche E-G, Gouvernet J, Azorin J-M, Akiskal HS. The cyclothymic temperament in healthy controls and familially at risk individuals for mood disorder: endophenotype for genetic studies? $J$ Affect Disord. 2005;85:135-45.

33. Hirschfeld RM, Calabrese JR, Frye MA, Lavori PW, Sachs G, Thase ME, Wagner KD. Defining the clinical course of bipolar disorder: response, remission, relapse, recurrence, and roughening. Psychopharmacol Bull. 2007;40:7-14.

34. Keller MB. Past, present, and future directions for defining optimal treatment outcome in depression: remission and beyond. JAMA. 2003;289:3152-60.

35. Tohen M, Frank E, Bowden CL, Colom F, Ghaemi SN, Yatham LN, Malhi GS, Calabrese JR, Nolen WA, Vieta E, Kapczinski F, Goodwin GM, Suppes T, Sachs GS, Chengappa KR, Grunze H, Mitchell PB, Kanba S, Berk M. The International Society for Bipolar Disorders (ISBD) Task Force report on the nomenclature of course and outcome in bipolar disorders. Bipolar Disord. 2009;11:453-73.

36. Zimmerman M, Mcglinchey JB, Posternak MA, Friedman M, Boerescu $\mathrm{D}$, Attiullah $\mathrm{N}$. Differences between minimally depressed patients who do and do not consider themselves to be in remission. $J$ Clin Psychiatry. 2005;66:1134-8.

37. Israel JA. Remission in depression: definition and initial treatment approaches. J Psychopharmacol. 2006;20:5-10.

38. Maremmani I, Akiskal HS, Signoretta S, Liguori A, Perugi G, Cloninger $R$. The relationship of Kraepelian affective temperaments (as 
measured by TEMPS-I) to the tridimensional personality questionnaire (TPQ). J Affect Disord. 2005;85:17-27.

39. Gurpegui D, OrtuñO F, Gurpegui M. Temperament traits and remission of depression: a naturalistic six-month longitudinal study. $J$ Affect Disord. 2019;243:494-502.

40. Ghaemi SN. Clinical psychopharmacology: principles and practice. Oxford: Oxford University Press; 2019.

41. Folstein MF, Folstein SE, McHugh PR. "Mini-mental state". A practical method for grading the cognitive state of patients for the clinician. $J$ Psychiatr Res. 1975;12:189-98.

42. Aprahamian I, Martinelli JE, Cecato J, Yassuda MS. Screening for alzheimer's disease among illiterate elderly: accuracy analysis for multiple instruments. J Alzheimers Dis. 2011;26:221-9.

43. Hamilton M. The assessment of anxiety states by rating. Br J Med Psychol. 1959;32:50-5.

44. Hamilton M. A rating scale for depression. J Neurol Neurosurg Psychiatry. 1960;23:56-62.

45. Vilela JAA, Crippa JAS, Del-Ben CM, Loureiro SR. Reliability and validity of a Portuguese version of the Young Mania Rating Scale. Braz J Med Biol Res. 2005;38:1429-39.

46. Woodruff E, Genaro LT, Landeira-Fernandez J, Cheniaux E, Laks J, Jean-Louis G, Nardi AE, Versiani MC, Akiskal HS, Mendlowicz MV. Validation of the Brazilian brief version of the temperament autoquestionnaire TEMPS-A: the brief TEMPS-Rio de Janeiro. J Affect Disord. 2011;134:65-76.

47. Karam EG, Hantouche E, Mneimneh Z. Temperament : where do you set the cutoff? AoA. 2006;2:165-9.

48. R Development Core Team. $R$ : A language and environment for statistical computing. R Foundation for Statistical Computing, Vienna, Austria. [Internet]. 2013. Available from: http://www.R-project.org/.

49. Cummings $P$, Rivara FP. Reporting statistical information in medical journal articles. Arch Pediatr Adolesc Med. 2003;157:321-4.

50. Ghaemi SN. A clinician's guide to statistics and epidemiology in mental health: measuring truth and uncertainty. Cambridge: Cambridge University Press; 2009.

51. Perugi G, Cesari D, Vannucchi G, Maccariello G, Barbuti M, De Bartolomeis A, Fagiolini A, Maina G. The impact of affective 
temperaments on clinical and functional outcome of Bipolar I patients that initiated or changed pharmacological treatment for mania.

Psychiatry Res. 2018;261:473-80.

52. Maina G, Salvi V, Rosso G, Bogetto F. Cyclothymic temperament and major depressive disorder: a study on Italian patients. J Affect Disord. 2010;121:199-203.

53. Terracciano A, Löckenhoff CE, Zonderman AB, Ferrucci L, Costa PT Jr. Personality predictors of longevity: activity, emotional stability, and conscientiousness. Psychosom Med. 2008;70:621-7.

54. Goto S, Terao T, Hoaki N, Wang Y. Cyclothymic and hyperthymic temperaments may predict bipolarity in major depressive disorder: A supportive evidence for bipolar II1/2 and IV. J Affect Disord. 2011;129:34-8.

55. de Aguiar Ferreira A, Vasconcelos AG, Neves FS, Correa H. Affective temperaments and antidepressant response in the clinical management of mood disorders. J Affect Disord. 2014;155:138-41.

56. Karam EG, Itani L, Fayyad J, Hantouche E, Karam A, Mneimneh Z, Akiskal H, Rihmer Z. Temperament and suicide: a national study. $J$ Affect Disord. 2015;184:123-8.

57. Baldessarini RJ, Vázquez GH, Tondo L. Affective temperaments and suicidal ideation and behavior in mood and anxiety disorder patients. $J$ Affect Disord. 2016;198:78-82.

58. Sudbrack R, Manfro PH, Kuhn IM, de Carvalho HW, Lara DR. What doesn't kill you makes you stronger and weaker: how childhood trauma relates to temperament traits. $J$ Psychiatr Res. 2015;62:123-9.

59. Kesebir S, Ünübol B, Tatlıdil Yaylacı E, Gündoğar D, Ünübol H. Impact of childhood trauma and affective temperament on resilience in bipolar disorder. Int J Bipolar Disord. 2015;3:3.

60. Toda H, Inoue T, Tanichi M, Saito T, Nakagawa S, Masuya J, Tanabe $\mathrm{H}$, Yoshino A, Kusumi I. Affective temperaments play an important role in the relationship between child abuse and the diagnosis of bipolar disorder. Psychiatry Res. 2018;262:13-9.

61. Kesebir S, Gündoğar D, Küçüksubaşı Y, Tatlıdil Yaylacı E. The relation between affective temperament and resilience in depression: a controlled study. J Affect Disord. 2013;148:352-6.

62. Dolenc B, Dernovšek MZ, Sprah L, Tavcar R, Perugi G, Akiskal HS. Relationship between affective temperaments and aggression in euthymic patients with bipolar mood disorder and major depressive disorder. J Affect Disord. 2015;174:13-8. 
63. Masi G, Milone A, Montesanto AR, Valente E, Pisano S. Non suicidal self-injury in referred adolescents with mood disorders and its association with cyclothymic-hypersensitive temperament. J Affect Disord. 2018;227:477-82. 